\title{
Combined T1-mapping and tissue tracking analysis predicts severity of ischemic injury following acute STEMI—an Oxford Acute Myocardial Infarction (OxAMI) study
}

\author{
Malgorzata Wamil ${ }^{1,2} \cdot$ Alessandra Borlotti ${ }^{1,2} \cdot$ Dan Liu $^{1,2} \cdot$ André Briosa e Gala $^{1,2} \cdot$ Alessia Bracco ${ }^{1,2}$. \\ Mohammad Alkhaliil ${ }^{1,2}$. Giovanni Luigi De Maria ${ }^{1,2}$. Stefan K. Piechnik ${ }^{1,2}$. Vanessa M. Ferreira ${ }^{1,2}$. \\ Adrian P. Banning ${ }^{1,2} \cdot$ Rajesh K. Kharbanda ${ }^{1,2} \cdot$ Stefan Neubauer ${ }^{1,2} \cdot$ Robin P. Choudhury $^{1,2} \cdot$ Keith M. Channon ${ }^{1,2}$. \\ Erica Dall'Armellina ${ }^{3,4}$
}

Received: 9 November 2018 / Accepted: 18 January 2019 / Published online: 16 February 2019

(c) The Author(s) 2019

\begin{abstract}
Early risk stratification after ST-segment-elevation myocardial infarction (STEMI) is of major clinical importance. Strain quantifies myocardial deformation and can demonstrate abnormal global and segmental myocardial function in acute ischaemia. Native T1-mapping allows assessment of the severity of acute ischemic injury, however its clinical applicability early post MI is limited by the complex dynamic changes happening in the myocardium post MI. We aimed to explore relationship between T1-mapping and feature tracking imaging, to establish whether combined analysis of these parameters could predict recovery after STEMI. 96 STEMI patients (aged 60 \pm 11 ) prospectively recruited in the Oxford Acute Myocardial Infarction (OxAMI) study underwent 3T-CMR scans acutely (within $53 \pm 32 \mathrm{~h}$ from primary percutaneous coronary intervention) and at 6 months $(6 \mathrm{M})$. The imaging protocol included: cine, ShMOLLI T1-mapping and late gadolinium enhancement (LGE). Segments were divided in the infarct, adjacent and remote zones based on the presence of LGE. Peak circumferential (Ecc) and radial (Err) strain was assessed using cvi42 software. Acute segmental strain correlated with segmental T1-mapping values (T1 vs. Err $-0.75 \pm 0.25, \mathrm{p}<0.01$; T1 vs. Ecc $0.72 \pm 0.32, \mathrm{p}<0.01$ ) and with LGE segmental injury (LGE vs. Err $-0.56 \pm 0.29, p<0.01 ;$ LGE vs. Ecc $0.54 \pm 0.35, p<0.01)$. Moreover, acute segmental $\mathrm{T} 1$ and strain predicted segmental LGE transmurality on $6 \mathrm{M}$ scans $(\mathrm{p}<0.001, \mathrm{r}=0.5)$. Multiple regression analysis confirmed combined analysis of global Ecc and T1-mapping was significantly better than either method alone in predicting final infarct size at $6 \mathrm{M}(\mathrm{r}=0.556 \mathrm{vs} r=0.473$ for global T1 only and $\mathrm{r}=0.476$ for global Ecc only, $\mathrm{p}<0.001$ ). This novel CMR method combining T1-mapping and feature tracking analysis of acute CMR scans predicts LGE transmurality and infarct size at 6M following STEMI.
\end{abstract}

Keywords Strain $\cdot$ Myocardial infarction $\cdot$ Cardiac magnetic resonance

$\begin{array}{ll}\text { Abbreviations } \\ \text { MI } & \text { Myocardial infarction } \\ \text { STEMI } & \text { ST-segment elevation myocardial infarction } \\ \text { PPCI } & \text { Primary percutaneous intervention } \\ \text { LGE } & \text { Late gadolinium enhancement } \\ \text { CASTS } & \text { The combined acute segmental native } \\ & \text { T1-mapping and the strain analysis } \\ \text { Ecc } & \text { Circumferential strain } \\ \text { Err } & \text { Radial strain } \\ \text { EF } & \text { Ejection fraction }\end{array}$

Erica Dall'Armellina

E.DallArmellina@leeds.ac.uk

Extended author information available on the last page of the article

$\begin{array}{ll}\text { T2W } & \text { T2Weighted images } \\ \text { ECV } & \text { Myocardial extracellular volume } \\ \text { FTI } & \text { Feature tracking imaging } \\ \text { GRE_PSIR } & \begin{array}{l}\text { T1-weighted segmented inversion recovery } \\ \text { gradient echo-phase sensitive-inversion } \\ \end{array} \\ & \begin{array}{l}\text { recovery } \\ \text { Steady state free precession }\end{array}\end{array}$

\section{Introduction}

Myocardial infarct (MI) size is a major determinant of the left ventricular (LV) dysfunction and long term remodelling in patients post ST-segment-elevation myocardial infarction (STEMI) [1]. Although primary percutaneous 
coronary intervention (PPCI) revolutionised the management of acute MI patients by allowing early intervention and saving myocardium from irreversible injury, there is still considerable and partially unexplained variability in the long-term outcome [2]. Therefore, accurate early risk stratification is of major clinical importance. Despite the broad use of LV ejection fraction (EF) in assessing post-MI LV remodelling, its prognostic value is poor [3]. Standard cardiovascular magnetic resonance (CMR) tissue characterization techniques such as late gadolinium enhancement (LGE) and T2Weighted (T2W) imaging provide several markers of long-term prognosis and remodelling [4]. Despite the availability of several highly accurate imaging markers (such as infarct size, transmurality, salvage myocardium), there is yet no definite consensus on the most relevant parameter. Additionally, novel CMR findings arising from imaging in the first hours post ischemia [5], seem to suggest the unfolding of complex dynamic tissue changes [6,7] hampering the prognostic relevance of standard LGE techniques [8,9] and indicating the need for more accurate techniques for stratification of patients early after MI.

By allowing quantitative assessment of the severity of the ischemic injury on a continuous scale, CMR mapping techniques allow accurate characterisation of the composition and viability of the myocardium after MI [10-12] providing additional predictive markers of remodelling and mortality in the infarcted core [13], and remote myocardium [14-16]. The early longitudinal changes happening in the myocardium, limit the ability to define the clinical significance of the severity of the myocardial injury earlier than $72 \mathrm{~h}$. A marker defining the functional impact of such changes and its effects on late remodelling is needed. Post ischemia, the changes happening in the tissue composition lead to impairment in myocardial deformation [17]. Historically, tagging was used to assess myocardial deformation by CMR, however, the applicability of such a technique in acute MI patients is challenging due to long scan times, breath holds and lengthy post processing [18]. Another recently validated CMR method, feature tracking imaging (FTI), allows measurements of circumferential, radial and longitudinal myocardial strain by tracking tissue voxel motion in cine CMR images avoiding the acquisition of additional sequences [19-22]. Whilst the incremental prognostic value of FTI in MI patients has been recently demonstrated above EF and infarct size [23], the relationship between myocardial strain and markers of severity of ischemic injury, as assessed by native quantitative mapping techniques, has not yet been fully defined. Here, we sought to investigate the additional predictive value of clinical outcome for markers of acute myocardial deformation such as circumferential (Ecc) and radial strain (Err) by FTI, to T1-mapping tissue characterization in STEMI patients.

\section{Methods}

\section{Patient population}

96 STEMI patients who underwent PPCI were prospectively enrolled in the study at the John Radcliffe Hospital as part of the OxAMI Study. This was a pre-specified study within the OxAMI research programme. Patients were eligible if the onset of symptoms had been within $12 \mathrm{~h}$ before PPCI and if they had ST-segment elevation of at least $0.1 \mathrm{mV}$ in $\geq 2$ contiguous limb leads or at least $0.2 \mathrm{mV} \geq 2$ contiguous precordial leads. Patients with previous MI, previous revascularization procedure (coronary artery bypass grafts or PCI), severe heart valve disease, known cardiomyopathy, or hemodynamic instability lasting $\geq 12 \mathrm{~h}$ after revascularization were excluded. Further exclusion criteria were contraindications to CMR, including implanted pacemakers, defibrillators, or other metallic implanted devices and claustrophobia. Acute clinical management was at the discretion of the responsible physician, with the intention to reflect contemporary practice and guidelines. The study protocol was approved by the local ethics committee, and all patients gave written informed consent.

\section{Cardiac magnetic resonance}

Patients underwent 3 T CMR scan (either MAGNETOM TIM Trio or MAGNETOM Verio, Siemens Healthcare, Erlangen, Germany) at 2 time points: acutely (within $53 \pm 32 \mathrm{~h}$ post PPCI) and at 6 months $(6 \mathrm{M})$. Matching short axis slices covering the LV were acquired using an established CMR protocol including: cine, native T1-mapping using the Shortened Modified Look-Locker Inversion recovery (ShMOLLI), and LGE as described in the Table 1 [24]. ShMOLLI T1 maps were generated from 5 to 7 SSFP images with variable inversion preparation time as described previously [24, 25]. Briefly, typical acquisition parameters were: TE/TR $=1.07 / 2.14 \mathrm{~ms}$, flip angle $=35^{\circ}, \mathrm{FOV}=340 \times 255 \mathrm{~mm}$, matrix size $=192 \times 144$, 107 phase encoding steps, actual experimental voxel size $=1.8 \times 1.8 \times 8 \mathrm{~mm}$, interpolated reconstructed voxel size $=0.9 \times 0.9 \times 8 \mathrm{~mm}$, GRAPPA $=2,24$ reference lines, cardiac delay time $\mathrm{TD}=\mathrm{S} 2500 \mathrm{~ms}$ and $206 \mathrm{~ms}$ acquisition time for single image, phase partial Fourier 6/8. LGE was performed with a T1-weighted segmented inversion recovery gradient echo-phase sensitive-inversion recovery (GRE_PSIR) sequence (TE/TR $=2.5 \mathrm{~ms} / 5 \mathrm{~ms}$, voxel size $1.8 \times 1.4 \times 8 \mathrm{~mm}$, flip angle $\left.20^{\circ}\right)$. LGE were collected $10-15 \mathrm{~min}$ after the administration of $0.1 \mathrm{mmol} / \mathrm{kg}$ contrast agent (Gadodiamide, Omniscan TM, GE Healthcare, 
Table 1 Characteristics of CMR sequences

\begin{tabular}{llll}
\hline $\begin{array}{l}\text { Imaging method } \\
\text { Sequence }\end{array}$ & $\begin{array}{l}\text { Cine } \\
\text { SSFP }\end{array}$ & $\begin{array}{l}\text { ShMOLLI T1 maps } \\
\text { 5-7 SSFP images }\end{array}$ & $\begin{array}{l}\text { LGE } \\
\text { T1-weighted GRE_PSIR }\end{array}$ \\
\hline TR, ms & 3.2 & 2.14 & 5 \\
TE, ms & 1.4 & 1.07 & 2.5 \\
Flip angle & $50^{\circ}$ & $35^{\circ}$ & $20^{\circ}$ \\
In-plane resolution & $1.6 \times 1.6$ & $1.8 \times 1.8$ & $1.8 \times 1.4$ \\
Slice thickness, mm & 8 & 8 & 8 \\
Other parameters & & & Optimal T1 to null the \\
& & & remote myocardium \\
\hline
\end{tabular}

GRE_PSIR gradient echo-phase sensitive-inversion recovery sequence; SSFP steady-state free precession sequence
Amersham, UK). The inversion time was adjusted for optimal nulling of remote normal myocardium. SSFP cine images were acquired using retrospective gating (TE/ $\mathrm{TR}=1.4 / 3.2 \mathrm{~ms}$; flip angle $=50^{\circ}$; pixel size: $\left.1.6 \times 1.6 \mathrm{~mm}\right)$. Two to three-fold accelerated parallel imaging (GRAPPA) was used to shorten the breath-hold on LGE imaging.

\section{CMR imaging analysis}

\section{Global analysis}

Anonymised images were analysed using cvi42 software (Circle Cardiovascular Imaging Inc., Calgary, Canada) by two experienced operators ( $\mathrm{MW}$ and $\mathrm{AB}$; both $>5$ years of CMR analysis experience) blinded to CMR measurements of the same patient across two time points and included LV volumes, quantification of LV\% oedema on T1 maps [26], LV\% necrosis/scar on LGE, global, circumferential and radial strain as previously described [22, 27]. The LGE signal intensity threshold for quantification of necrosis/scar was set at 5 standard deviations (SD) above the reference ROI in the remote unaffected myocardium [26, 28]. Global mean T1-mapping values were calculated averaging segmental T1-mapping values per patient. The final infarct size was defined as the LV\% LGE at 6M.

\section{Segmental analysis}

Segmental analysis was performed on the short-axis (SA) images (Fig. 1). For all the techniques SA images were divided in six equiangular segments with the anterior $\mathrm{RV}-\mathrm{LV}$ junction as reference point. The following segmental measurements were assessed: LGE percentage fraction, peak radial (Err) and circumferential (Ecc) strain (\%), native T1 values. Segmental LGE was calculated as percentage fraction of the segment volume. Segmental peak radial (Err) and circumferential (Ecc) strain (\%) were analysed based on the previously published methods [29] and were calculated using 50 cords at end-systole and end-diastole using the same, standard epicardial and endocardial contours as drawn for diastolic and systolic volume analysis [30]. Endoand epicardial contours were drawn manually in all slices by one skilled observer. T1 maps underwent strict and extensive quality control as previously described [24]. Segmental T1 values were derived from short-axis T1 maps using in-house dedicated software MC-ROI (Interactive Data Language, version 6.1, Exelis Visual Information Solutions, Boulder, Colorado). Segments with MVO were excluded from the analysis. Far apical slices with partial volume effects and slices with visible outflow tracts were excluded from the study.

Cvi42 software was used to assess LGE transmurality (Tissue characterisation: LGE module). Briefly, contours were drawn manually around LV endo- and epicardium. The LGE signal intensity threshold for quantification of necrosis was set at five standard deviations (SD) above the reference ROI in the remote unaffected myocardium [26, 28]. Myocardium was divided into six standard segments and LGE transmurality was reported per segment. Segments were graded according to peak LGE transmurality in end-diastole as follows (1)>0 to $\leq 25 \%(2)>25$ to $\leq 50 \%$ (3) $>50$ to $\leq 75 \%$ and (4) $>75 \%$. Segments showing LGE $>25 \%$ were defined as infarcted, whilst segments with no LGE and located 180 degrees from the infarct, were defined as remote, as previously described [31]. Segments located on the same plane contiguously to the infarcted segments and with LGE $<25 \%$ were defined as adjacent (Fig. 2). Infarcted segments with persistent $\mathrm{LGE} \geq 50 \%$ at $6 \mathrm{M}$ scans were identified as irreversibly damaged. Segments within the infarct zone with LGE $\geq 25 \%$ on the acute scan and negative LGE on $6 \mathrm{M}$ scan were identified as recovered segments.

\section{Statistical analysis}

Statistical analyses were performed using IBM SPSS Statistics (version 22.0, IBM Corporation, Armonk, NY). Continuous data are expressed as mean \pm SD except where otherwise specified. Categorical variable was expressed as $\mathrm{n} \%$. The 

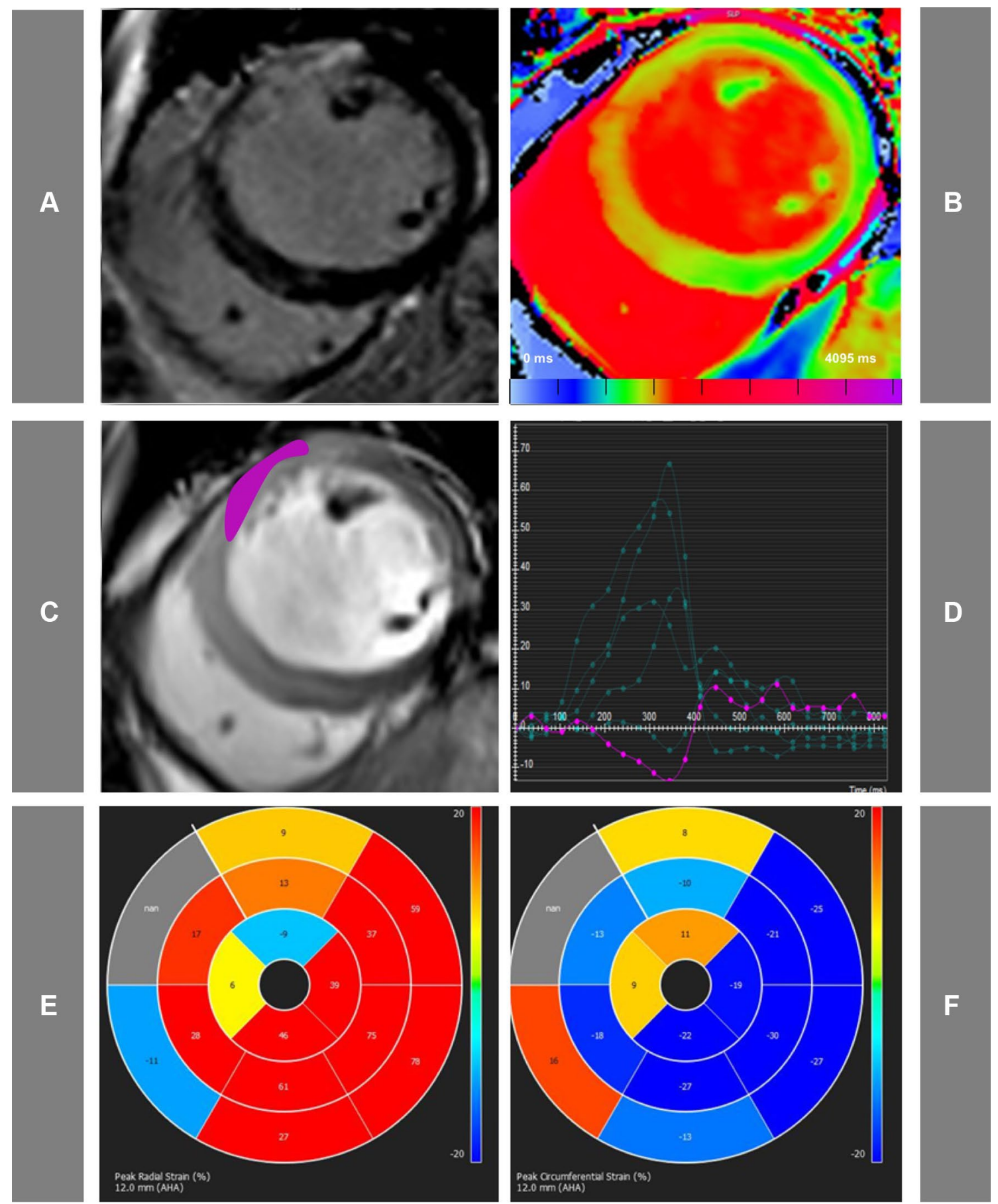

Fig. 1 Representative acute CMR images used for the assessment of STEMI patients. Matching mid ventricular short axis slices acquired using LGE PSIR images (a), native T1-mapping (b), functional cine imaging (c). d The cvi42-derived tissue tracking analysis, whilst $\mathbf{e}$ and $\mathbf{f}$ display representative bull's eye maps for radial and

normality of the data was assessed using the Shapiro-Wilk test or the Kolmogorov-Smirnov test depending on the size of samples tested. Those variables that did not follow a normal distribution are presented as medians with interquartile circumferential strain assessment, respectively. In this case of acute anterior myocardial infarction, the myocardium co-localized with the enhanced areas on LGE images, show prolonged T1 values and abnormal peak radial (Err) and circumferential (Ecc) strain

range (median (IQR)). Comparisons between groups were performed with t-test or one-way ANOVA where normality was met or with the non-parametric Kruskal-Wallis H. Test when data normality was violated (differences in median $\mathrm{T} 1$ 


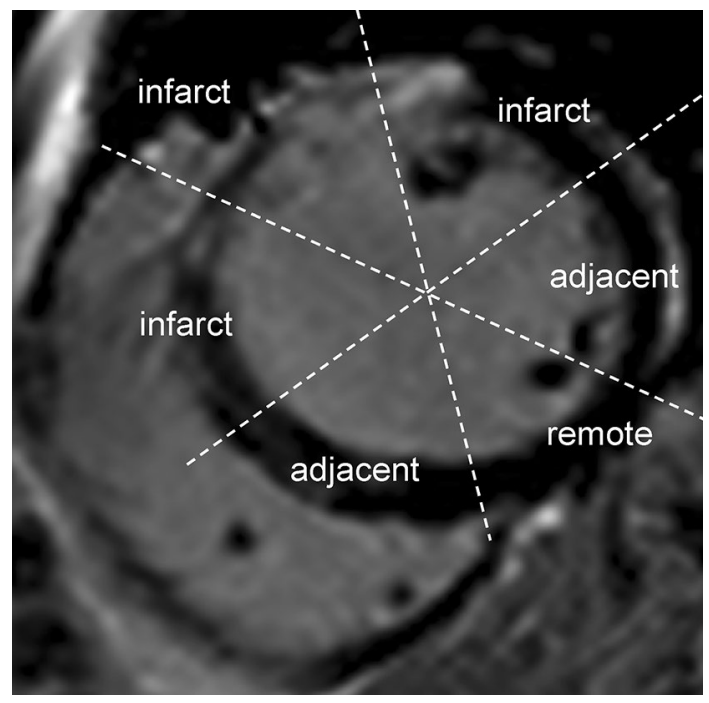

Fig. 2 Definition of the infarcted and the adjacent myocardium. This is a representative short axis image of a patient with anterior myocardial infarction by late gadolinium enhancement. Segments with LGE $>25 \%$ were identified as infarcted, whilst segments located on the same plane contiguously to the infarcted segments and with LGE $<25 \%$ were defined as adjacent; myocardium with no LGE and $180^{\circ}$ from the infarct was defined as remote

values, LGE, Err and Ecc in infarct, adjacent and remote segments). Correlation was expressed as Pearson or Spearman correlation coefficient. To account for the fact that segmental $\mathrm{T} 1$ values and segmental peak strain analysis could not be treated as independent samples, the following approach was utilised: correlation coefficient values from the segmental analysis of two imaging biomarkers was first derived for each patient, then transformed with the Fisher's z' transformation that converts Pearson's r's to the normally distributed variable $\mathrm{z}$ and subsequently analysed by t-test [32]. CASTS (combined analysis of segmental native T1-mapping and segmental tissue tracking analysis) is a term, which we used for the combined acute segmental strain and acute T1-mapping analysis and was derived from logistic regression and then tested as predictive probability against strain and T1-mapping values separately. The linear regression was used to assess the performance of the combined native T1-mapping and strain analysis in predicting the final scar size at $6 \mathrm{M}$ time. Multiple regression analysis was used to test the contribution of T1 mapping, Ecc and Err in predicting $6 \mathrm{M}$ peak LGE transmurality. P-values less than 0.05 were considered statistically significant.

\section{Results}

Patient characteristics are given in Table 2. One hundred and twenty patients were recruited of which 24 were excluded due to the following reasons: claustrophobia $(n=10)$,
Table 2 Baseline characteristics of the study population

\begin{tabular}{ll}
\hline & Mean \pm SD \\
\hline Age, year & $60 \pm 11$ \\
Sex, M/F & $68 / 28$ \\
Risk factors, n (\%) & \\
Diabetes & $10(10)$ \\
Smoker & $26(27)$ \\
Hypertension & $33(34)$ \\
Hyperlipidaemia & $34(35)$ \\
Family history of CHD & $35(36)$ \\
Target vessel n (\%) & \\
LAD & $43(45)$ \\
LCx & $7(7)$ \\
RCA & $26(27)$ \\
Peak troponin (mg/l) & $206 \pm 290$ \\
Pain to Balloon time (mins) & $242 \pm 180$ \\
PPCI to CMR time (h) & $53 \pm 36$ \\
No. of vessels diseased, n (\%) & \\
1 & $72(75)$ \\
2 & $17(19)$ \\
3 & $7(6)$ \\
TIMI flow pre PCI, n (\%) & $10(12)$ \\
0 & $1(1)$ \\
2 & $66(80)$ \\
TIMI flow post PCI, n (\%) & $5(6)$ \\
2 & $68(93)$ \\
\hline & \\
&
\end{tabular}

LAD indicates left anterior descending artery, LCx left circumflex artery, RCA right coronary artery

scanner failure $(\mathrm{n}=2)$ and poor CMR image quality (due to breathing artefacts on acute T1 maps or LGE images) $(\mathrm{n}=12)$. Out of the remaining 96 STEMI patients with acute CMR ( $53 \pm 36 \mathrm{~h}$ post $\mathrm{PCI}), 59$ patients underwent a $6 \mathrm{M}$ follow up scan. Reasons for not returning for follow-up CMR scans were as follows: withdrawal from the study $(n=30)$, death $(n=3)$, and implantable cardioverter-defibrillator $(n=4)$.

\section{CMR findings}

CMR findings are summarized in Table 3. On LGE images, only one patient had no evidence of enhancement. At $6 \mathrm{M}$, EF improved by $5 \%(\mathrm{p}<0.001)$ (Table 3$)$. There was a significant correlation between global strain and global native T1-mapping: T1 versus Err $\mathrm{r}=-0.495, \mathrm{p}<0.001$, T1 versus Ecc $r=-0.571, p<0.001$ (Fig. 3). 
Table 3 CMR findings

\begin{tabular}{lccc}
\hline & $\begin{array}{l}\text { Acute mean } \pm \text { SD } \\
(\mathrm{n}=96)\end{array}$ & $\begin{array}{l}6 \mathrm{M} \text { mean } \pm \text { SD } \\
(\mathrm{n}=59)\end{array}$ & $p$-Value \\
\hline EF (\%) & $46 \pm 8$ & $51 \pm 9$ & $<0.001$ \\
EDV (ml) & $164 \pm 43$ & $163 \pm 39$ & 0.9 \\
ESV (ml) & $89 \pm 33$ & $78 \pm 31$ & $<0.01$ \\
SV (ml) & $73 \pm 20$ & $86 \pm 18$ & $<0.01$ \\
Oedema by T1-mapping, (\%LV) & $42 \pm 14$ & $16 \pm 10$ & $<0.001$ \\
LGE, (\%LV) & $24 \pm 14$ & & \\
Myocardial salvage index & $47 \pm 22$ & \\
[(Oedema\%LV-\%V LGE)/Oedema \%LV] & $2 \pm 3$ & $1214 \pm 75$ & $<0.001$ \\
MVO (\%LV) & $1280 \pm 47$ & & \\
Global LV T1 (ms) & $-16.6 \pm 3.8$ & \\
Global circumferential strain (\%) & $29.6 \pm 9$ & \\
Global radial strain (\%) & & \\
\hline
\end{tabular}

$E F$ ejection fraction, $E D V$ end-diastolic volume, $E S V$ end-systolic volume, $L G E$ late gadolinium enhancement, $L V$ left ventricle, $M V O$ microvascular obstruction

Fig. 3 Correlation between global native T1-mapping and global circumferential strain. Global analysis of averaged $\mathrm{T} 1$ values (calculated as averaged values per patient) and global circumferential strain analysis (derived from tissue tracking analysis cvi42 software) showed a significant correlation $(\mathrm{r}=0.571, \mathrm{p}<0.001)$

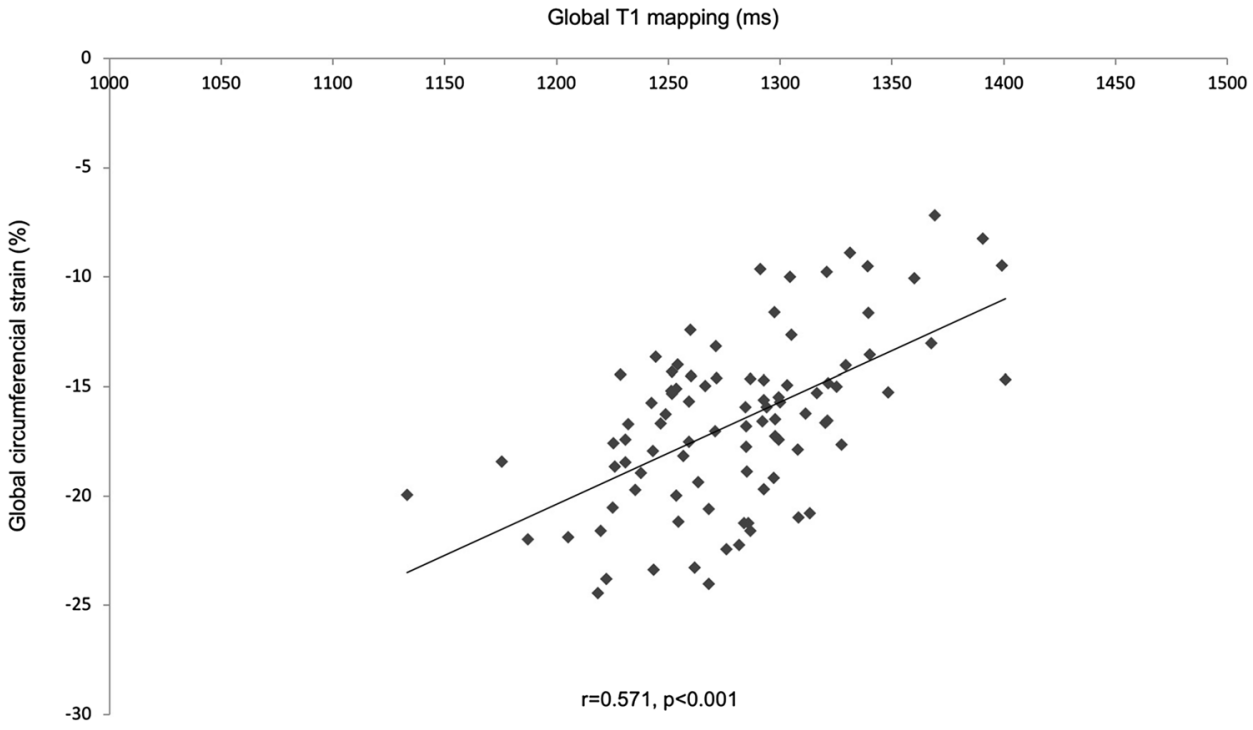

\section{Segmental analysis}

A total of 3415 acute segments and 1474 on the $6 \mathrm{M}$ scans were suitable for analysis. Out of all acute segments, there were 90 segments with $\mathrm{LGE}=100 \%, 231$ segments with LGE $\geq 75 \%$ and $<100 \%, 319$ segments with LGE $\geq 50 \%$ and $<75 \%$ and 410 segments with LGE $>25 \%$ and $<50 \%$ (Fig. 4). As shown in Table 4, acute native T1 values in infarcted and adjacent segments were significantly prolonged compared to remote myocardium $(1357 \pm 89 \mathrm{~ms}$ infarcted vs. $1245 \pm 100 \mathrm{~ms}$ adjacent vs. $1215 \pm 87 \mathrm{~ms}$, remote, $\mathrm{p}<0.001)$. Acutely, 20\% of the remote segments (293/1434) showed significantly prolonged T1 values comparing to normal values [33] $(1229 \pm 76 \mathrm{~ms}$ vs. $1180 \pm 36 \mathrm{~ms}, \mathrm{p}<0.001)$. Native $\mathrm{T} 1$ values recovered significantly over time in infarcted, adjacent myocardium and also in those remote segments showing prolongation of T1 acutely (infarct: acute scan $1357 \pm 89$ ms vs. $6 \mathrm{M}$ scan $1235 \pm 99 \mathrm{~ms}, \mathrm{p}<0.01$; adjacent zones: acute scan $1245 \pm 100$ ms vs. $6 \mathrm{M}$ scan $1193 \pm 66$, ms $\mathrm{p}<0.01$; remote acute scan: $1215 \pm 87 \mathrm{~ms}$ vs. $6 \mathrm{M}$ scan $1185 \pm 66 \mathrm{~ms}, \mathrm{p}<0.01)$.

\section{Relationship between severity of ischemic injury and strain}

Both Err and Ecc worsened progressively as acute T1 values increased from remote to adjacent to infarcted myocardium (median (IQR) Err ${ }_{\text {infarct }}=12(13) \%$ vs. Err $_{\text {remote }}=35$ (23)\%, p < 0.01; $\mathrm{Ecc}_{\text {infarct }}=-10(9) \%$ vs. Ecc $_{\text {remote }}=-20$ (9)\%, p <0.01) (Table 5). The difference between Ecc in adjacent versus remote segments did not reach significance 


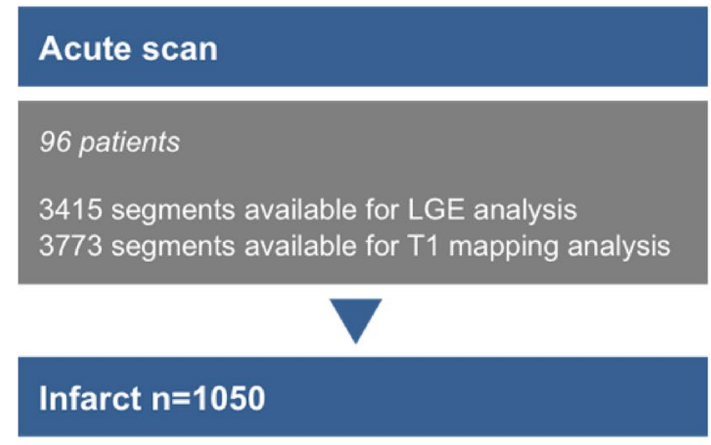

90 segments with LGE $=100 \%$

231 segments with LGE $\geq 75 \%$ and $<100 \%$

319 segments with LGE $\geq 50 \%$ and $<75 \%$

410 segments with LGE $>25 \%$ and $<50 \%$
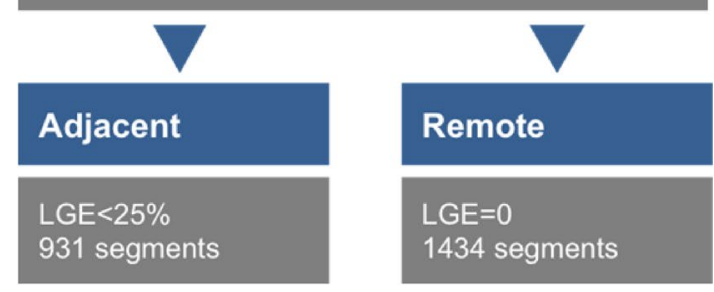

\section{$6 \mathrm{M}$ scan}

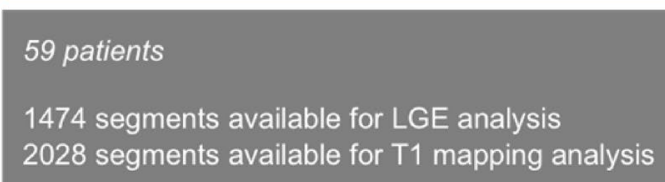

\section{Infarct $n=312$}

26 segments with LGE $=100 \%$

70 segments with LGE $\geq 75 \%$ and $<100 \%$

80 segments with LGE $\geq 50 \%$ and $<75 \%$

136 segments with LGE $>25 \%$ and $<50 \%$
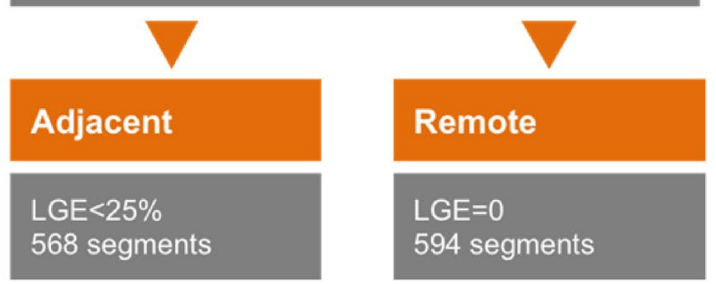

Fig. 4 The figure shows the distribution of segments in the study on the acute and 6M scans according to LGE transmurality. Segments were divided into infarct, adjacent and remote zones based on LGE transmurality and their location as described in the "Methods"

Table 4 CMR segmental values of strain and tissue injury acutely and at $6 \mathrm{M}$

\begin{tabular}{lllllll}
\hline & & $\begin{array}{l}\text { Infarct } \\
\mathrm{n}=1050\end{array}$ & $\begin{array}{l}\text { Adjacent } \\
\mathrm{n}=931\end{array}$ & $\begin{array}{l}\text { Remote } \\
\mathrm{n}=1434\end{array}$ & $\begin{array}{l}p \text { Value } \\
\text { (infarct vs. remote) }\end{array}$ & $\begin{array}{l}p \text { Value } \\
\text { (adja- } \\
\text { cent vs. } \\
\text { remote) }\end{array}$ \\
\hline Acute CMR & T1 (ms) & $1357 \pm 89$ & $1245 \pm 100$ & $1215 \pm 87$ & $<0.001$ & $<0.001$ \\
& LGE (\%) & $52 \pm 53$ & $0 \pm 6$ & $0 \pm 0$ & $<0.001$ & $<0.001$ \\
& Err (\%) & $12(13)$ & $27(19)$ & $35(23)$ & $<0.001$ & $<0.01$ \\
& Ecc (\%) & $-10(9)$ & $-17(8)$ & $-20(9)$ & $<0.001$ & 0.07 \\
6M CMR & T1 (ms) & $1235 \pm 99$ & $1193 \pm 66$ & $1185 \pm 66$ & $<0.001$ & 0.387 \\
& LGE (\%) & $36(56)$ & $0(5)$ & $0(0)$ & $<0.001$ & $<0.001$ \\
\hline
\end{tabular}

Data are expressed as mean $\pm \mathrm{SD}$ or median $(\mathrm{IQR})$

\begin{tabular}{|c|c|c|c|c|c|c|}
\hline & & $\begin{array}{l}\text { LGE }<25 \% \\
n=2538\end{array}$ & $\begin{array}{l}\text { LGE } 25-50 \% \\
\mathrm{n}=319\end{array}$ & $\begin{array}{l}\text { LGE } 50-75 \% \\
\mathrm{n}=225\end{array}$ & $\begin{array}{l}\text { LGE } \geq 75 \% \\
n=333\end{array}$ & $\begin{array}{l}p \text { Value } \\
\text { (difference } \\
\text { between } \\
\text { groups) }\end{array}$ \\
\hline \multirow[t]{3}{*}{ Acute CMR } & $\mathrm{T} 1$ (ms) & $1229 \pm 94$ & $1332 \pm 86$ & $1374 \pm 91$ & $1378 \pm 84$ & $<0.001$ \\
\hline & $\operatorname{Err}(\%)$ & $31.4(25)$ & $14(15)$ & $11(10)$ & $8.8(9)$ & $<0.001$ \\
\hline & $\operatorname{Ecc}(\%)$ & $-19(10)$ & $-12(10)$ & $-10(8)$ & $-7.5(7)$ & $<0.001$ \\
\hline 6M CMR & $\mathrm{T} 1$ (ms) & $1197 \pm 68$ & $1210 \pm 83$ & $1245 \pm 96$ & $1268 \pm 87$ & $<0.001$ \\
\hline $\begin{array}{l}p \text { Value (acute } \\
\text { vs. } 6 \mathrm{M} \mathrm{T} 1 \text { ) }\end{array}$ & & $<0.001$ & $<0.001$ & $<0.001$ & $<0.001$ & \\
\hline
\end{tabular}

Data are expressed as mean \pm SD or median (IQR)
Table 5 CMR segmental T1, Err and Ecc values according to LGE transmurality 
Table 6 Correlation coefficients between acute segmental native T1-mapping values and segmental radial (Err) and circumferential (Ecc) strain as well as LGE

\begin{tabular}{lllll}
\hline & T1 vs. Err & T1 vs. Ecc & LGE vs. Err & LGE vs. Ecc \\
\hline Mean \pm SD & $-0.75 \pm 0.25$ & $0.72 \pm 0.32$ & $-0.56 \pm 0.29$ & $0.54 \pm 0.35$ \\
p Value & $\mathrm{p}<0.001$ & $\mathrm{p}<0.001$ & $\mathrm{p}<0.001$ & $\mathrm{p}<0.001$ \\
n Patients & 96 & 96 & 96 & 96 \\
Segments & 3773 & 3773 & 3773 & 3773 \\
\hline
\end{tabular}

All segments were first analysed for correlations per patient $n=96$ and then these correlation coefficients were compared with one-sample t-test

$\left(\right.$ Ecc $_{\text {adjacent }}=-20(8) \%$ vs. Ecc remote $_{\text {zone }}-21$ (7)\%; $\mathrm{p}=0.07)$. Err and Ecc values varied significantly between segments grouped by LGE transmurality (Table 5). On a patient by patient basis, myocardial deformation by Err and Ecc correlated significantly with $\mathrm{T} 1$ values (T1 vs. Err $\mathrm{r}=-0.75 \pm 0.25, \mathrm{p}<0.01$; T1 vs. Ecc $\mathrm{r}=0.72 \pm 0.32$, $\mathrm{p}<0.01$ ) and with LGE segmental fraction (LGE vs. Err $r=-0.56 \pm 0.29, p<0.01 ;$ LGE vs. Ecc $r=0.54 \pm 0.35$, $\mathrm{p}<0.01$ ) (Table 6).

\section{The role of normal circumferential strain in the assessment of functional recovery of the adjacent segments}

Table 4 shows significantly prolonged native $\mathrm{T} 1$ values in the adjacent segments comparing to the infarct and remote zones. A trend towards a statistically significant difference in circumferential strain between adjacent and remote zones was shown (adjacent segments Ecc $-20 \pm 8 \%$ vs. remote zone $-21 \pm 7 \%, p=0.07$ ). There was no difference in the native $\mathrm{T} 1$ values in the remote and adjacent segments on the $6 \mathrm{M}$ scan suggesting a functional recovery of these segments.

\section{The additional value of combined acute segmental strain and T1-mapping (CASTS) for $6 \mathrm{M}$ prediction of LGE peak segmental transmurality}

In order to assess whether acute myocardial deformation in addition to myocardial composition, as assessed by $\mathrm{T} 1$ values, predict the $6 \mathrm{M}$ peak LGE segmental transmurality, we performed a multiple regression analysis including acute segmental T1, Err and Ecc values as independent variables. When combined these variables significantly predicted $6 \mathrm{M}$ segmental LGE transmurality $(\mathrm{p}<0.001, \mathrm{r}=0.5)$. Furthermore, we analysed the contribution of each of the independent variables to the prediction of the 6M peak LGE segmental transmurality. When $\mathrm{T} 1$ and Ecc values were used together both variables significantly improved the prediction of final segmental LGE transmurality $(\mathrm{p}<0.001$ for each of them), however, Err did not add to the prediction of $6 \mathrm{M}$ segmental LGE transmurality (Err $p=0.662)$. Thus, in further analyses we used T1-mapping combined with Ecc only.

\section{Predictive value of the combined analysis of global strain and T1-mapping in the assessment of $6 \mathrm{M}$ infarct size}

In view of the fact, that global rather than segmental stain has been previously shown to predict cardiac remodelling after STEMI [34], we wanted to examine the performance of global values obtained for the tissue tracking analysis and global $\mathrm{T} 1$ values (calculated as averaged values per patient). We then compared the combined predictive value of the global analysis of circumferential strain and global T1 values. We confirmed that combined analysis of these two biomarkers was a good predictor of the final infarct scar on the follow-up scan at $6 \mathrm{M}(\mathrm{p}<0.001, \mathrm{r}=0.556)$ and that both biomarkers when assessed globally improved significantly the prediction of the scar size (as assessed per patient as total LGE in \%; standardized coefficients for global T1 values 0.323, $\mathrm{p}=0.018$, for global Ecc 0.329, $\mathrm{p}=0.016$ ). Furthermore, we showed that combined analysis of these two global biomarkers was significantly outperforming global T1-mapping or global circumferential strain analysis alone $(r=0.556$ vs. $r=0.473$ for $\mathrm{T} 1$ only and $r=0.476$ for Ecc only; $\mathrm{p}<0.001)$.

\section{Discussion}

In patients with acute MI, novel CMR imaging markers derived from either tissue characterisations mapping techniques or FTI methods have been shown to have incremental prognostic value compared to standard LGE infarct size and $\mathrm{EF}[13,15,23]$. By determining voxel wise $\mathrm{T} 1$ values on a continuous scale as measurements of the tissue composition, T1-mapping provides additional information on the severity of injury allowing distinguishing between reversible and irreversible injury. The dynamic changes happening in the tissue composition during the healing phase post MI [35] could result in variations in the acute T1-mapping values [7]. Hence, there is a demonstrable need for an additional stable imaging parameter capable of increasing the accuracy of early risk stratification. Regional strain might be well suited to increase further the predictive value of mapping techniques by reflecting abnormal segmental myocardial deformation as a result of acute changes in tissue composition. By investigating the interrelationship between native T1-mapping and regional strain in acute MI, our study shows the additional value of the combined acute segmental strain and T1-mapping analysis (CASTS) in predicting irreversibility 


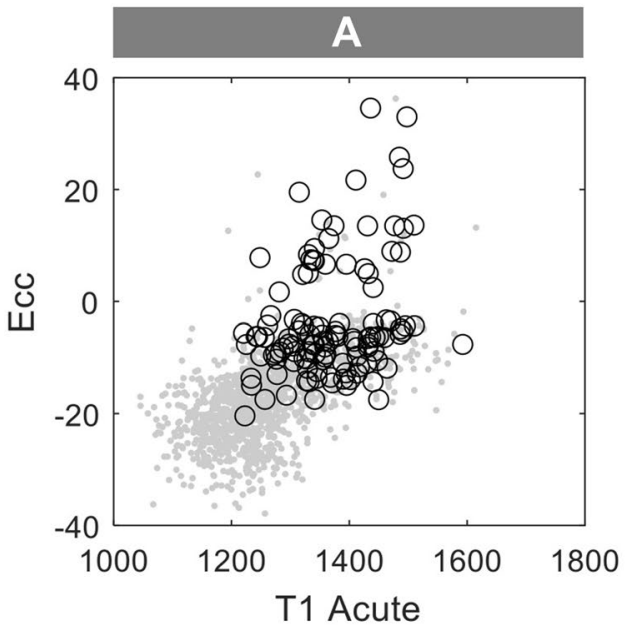

Fig. 5 Performance of the combined analysis of acute T1-mapping and circumferential strain (CASTS) for functional assessment postMI. Figure represents the performance of segmental native T1-mapping combined with segmental Ecc in identifying a irreversibly damaged segments visualised as black circles (> 50\% LGE on the acute

of the ischemic injury compared to either of these imaging markers alone (Fig. 5).

Whilst peak segmental strain and native T1-mapping analysis reliably identifies infarct and remote zones, in areas adjacent to the ischemic myocardium, circumferential strain measures did not significantly differ from remote myocardium despite of the high $\mathrm{T} 1$ values. This suggests that native T1-mapping and peak circumferential strain analysis combined is able to distinguish between infarcted, irreversibly damaged segments and peri-infarct zones that have a high chance to recover.

Moreover, we observed significant prolongation of the native $\mathrm{T} 1$ values in $20 \%$ of the non-ischaemic zones and their recovery on the follow-up scans. Significantly, all these segments had normal values of circumferential strain. This observation is particularly potent in light of gadolinium-free T1-mapping techniques potentially replacing contrast methods altogether. In our study, segments were divided in the infarct, adjacent and remote zones based on the presence of LGE. Thus, if one would rely exclusively on the analysis of segments of the native T1 values, these remote segments with increased T1-mapping values might get wrongly labeled as infarcted. Conversely, the addition of circumferential strain allows us to accurately identify these segments. This is particularly true when assessing zones adjacent to the infarct. Similar observations that were previously reported by Carrick et al. [14] and by Reinstadler et al. [15], who also showed increased native T1-mapping values in the nonischaemic myocardium post-MI. The authors confirmed that the remote native T1-mapping was independently predictive of MACE at 6 months after STEMI. They postulated

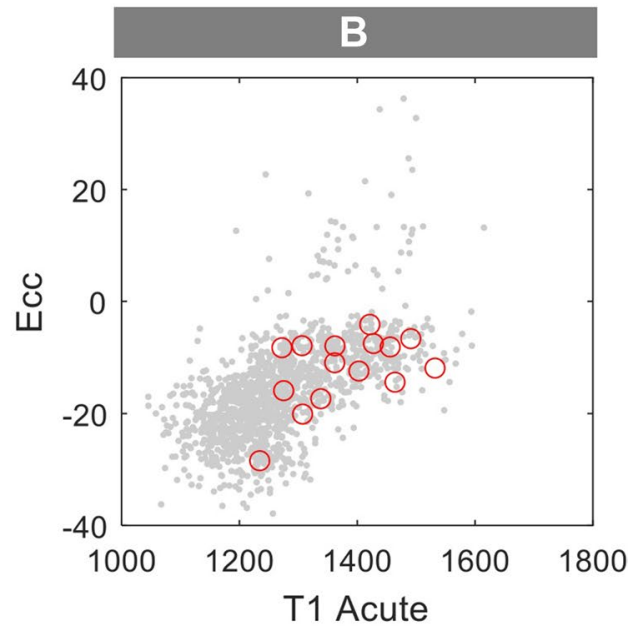

and $6 \mathrm{M}$ scan, segments with MVO were excluded from the analysis) and $\mathbf{b}$ normalised segments visualised as red circles (LGE $=0$ on the $6 \mathrm{M}$ scan and LGE $\geq 25 \%$ on the acute scan and in the area of infarction). Grey dots represent all assessed segments by these two methods

these alterations in the non-ischaemic zones $\mathrm{T} 1$ values were a result of inflammation and hypercellularity. Pre-clinical studies strongly support the presence of pro-inflammatory macrophages in the non-ischaemic remote myocardium in MI models [36]. Thus, we postulate that an increase in the remote zones $\mathrm{T} 1$ values acutely post-MI could reflect an exacerbated tissue inflammation affecting the non-ischaemic myocardium (remote and adjacent zones) early post-MI and that circumferential strain can help to distinguish these segments with high inflammatory changes but full chances to recover from those that were irreversibly damaged.

\section{Limitations}

The additive value of the combined analysis of segmental native T1 mapping and tissue tracking is somewhat smaller than anticipated. This could be explained by the fact that actually both imaging biomarkers measure the same process, namely acute injury caused by inflammation and oedema of the myocardium. Thus, changes in the tissue characteristic will influence changes in the tissue deformation measured by the peak strain. This observation is supported by our findings that show significant correlation between the percentage of global oedema and global strain.

Another important limitation of our study is a significant drop out in the follow-up CMR scans. 59 out of 96 recruited subjects returned for the $6 \mathrm{M}$ follow up scans. We decided to include all recruited patients in our study but we acknowledge the analysis of the predictive value of the combined analysis of global strain and T1-mapping in the assessment 
of $6 \mathrm{M}$ infarct size was performed on those subjects, which underwent full study protocol including $6 \mathrm{M}$ scans.

\section{Conclusions}

This novel CMR method combining T1-mapping and strain analysis of acute CMR scans improves the prediction of irreversible ischaemic injury following STEMI. Further studies using alternative mapping modalities and investigating the longitudinal changes over the early hours post MI, will be needed to establish a combined protocol of native T1 mapping and feature tracking assessment as an alternative to the standard LGE method, limiting the CMR protocol to noncontrast acquisitions.

Acknowledgements The authors thank the clinical staff in the Oxford Heart Centre and Oxford Centre for Clinical Magnetic Resonance Research (OCMR); Dr Paula Colmenero, Juliet Semple, Peter Manley, Carol Davey, and Lisa Gaughran in the Oxford Acute Vascular Imaging Centre and Prof Rafal Bogacz for help with statistical analysis.

Disclosures SKP has patent authorship rights for U.S. patent 9285446 B2. Systems and methods for shortened look locker inversion recovery (Sh-MOLLI) cardiac gated mapping of T1. Granted March 15, 2016. All rights transferred to Siemens Medical. All other authors have no relationships relevant to the contents of this paper to disclose.

Author contributions MW and EDA designed the project, analysed and interpreted the data and wrote the manuscript. KMC, APB, RKK, RPC, MA and GLDM recruited patients. DL, ABG and AB analysed the data. SKP, VMF, APB, RKK, SN, RPC and KMC reviewed the manuscript and provided intellectual input.

Funding Supported by the National Institute for Health Research (NIHR) Oxford Biomedical Research Centre and by the Oxford British Heart Foundation (BHF) Centre of Research Excellence (RG/13/1/30181); a BHF Intermediate Clinical Research Fellowship (to EDA), a BHF Oxford Centre of Research Excellence Clinical Research support (to MW, RC, KC, VMF, SKP and SN) and a BHF Chair Award (CH/16/1/32013, to KMC).

\section{Compliance with ethical standards}

Conflict of interest The authors of this manuscript declare no relationships with any companies, whose products or services may be related to the subject matter of the article.

Ethical approval REC reference 11/SC/0397 granted by the NRES Committee South Central-Oxford C on 29th of December 2011.

Informed consent Only if the study is on human subjects: written informed consent was obtained from all subjects (patients) in this study.

Open Access This article is distributed under the terms of the Creative Commons Attribution 4.0 International License (http://creativeco mmons.org/licenses/by/4.0/), which permits unrestricted use, distribution, and reproduction in any medium, provided you give appropriate credit to the original author(s) and the source, provide a link to the Creative Commons license, and indicate if changes were made.

\section{References}

1. Pfeffer MA, Braunwald E (1990) Ventricular remodeling after myocardial infarction. Experimental observations and clinical implications. Circulation 81(4):1161-1172

2. Yamashita Y, Shiomi H, Morimoto T, Yaku H, Furukawa Y, Nakagawa Y et al (2017) Cardiac and noncardiac causes of long-term mortality in ST-segment-elevation acute myocardial infarction patients who underwent primary percutaneous coronary intervention. Circ Cardiovasc Qual Outcomes 10(1):e002790

3. Dagres N, Hindricks G (2013) Risk stratification after myocardial infarction: is left ventricular ejection fraction enough to prevent sudden cardiac death? Eur Heart J 34(26):1964-1971

4. Grover S, Bell G, Lincoff M, Jeorg L, Madsen PL, Huang S et al (2015) Utility of CMR markers of myocardial injury in predicting LV functional recovery: results from PROTECTION AMI CMR sub-study. Heart Lung Circ 24(9):891-897

5. Dall'Armellina E (2016) From recognized to novel quantitative CMR biomarkers of LV recovery: a paradigm shift in acute myocardial infarction imaging. JACC Cardiovasc Imaging 10(9):1000-1002

6. Carrick D, Haig C, Ahmed N, Rauhalammi S, Clerfond G, Carberry J et al (2016) Temporal evolution of myocardial hemorrhage and edema in patients after acute ST-segment elevation myocardial infarction: pathophysiological insights and clinical implications. J Am Heart Assoc 5(2):e002854

7. Fernandez-Jimenez R, Sanchez-Gonzalez J, Aguero J, GarciaPrieto J, Lopez-Martin GJ, Garcia-Ruiz JM et al (2014) Myocardial edema after ischemia/reperfusion is not stable and follows a bimodal pattern: advanced imaging and histological tissue characterization. J Am Coll Cardiol 65(4):315-323

8. Matsumoto H, Matsuda T, Miyamoto K, Shimada T, Mikuri M, Hiraoka Y (2011) Peri-infarct zone on early contrast-enhanced CMR imaging in patients with acute myocardial infarction. JACC: Cardiovasc Imaging 4(6):610-618

9. Hammer-Hansen S, Leung SW, Hsu L-Y, Wilson JR, Taylor J, Greve AM et al (2017) Early gadolinium enhancement for determination of area at risk: a preclinical validation study. JACC: Cardiovasc Imaging 10(2):130-139

10. Yan G, Meng Z, Liu N, He L, Paterson KB (2017) Effects of irrelevant background speech on eye movements during reading. Q J Exp Psychol (Hove). https://doi.org/10.1080/17470 218.2017.1339718

11. Bulluck H, White SK, Rosmini S, Bhuva A, Treibel TA, Fontana $M$ et al (2015) T1 mapping and T2 mapping at 3T for quantifying the area-at-risk in reperfused STEMI patients. J Cardiovasc Magn Reson 17(1):73

12. Garg P, Broadbent DA, Swoboda PP, Foley JRJ, Fent GJ, Musa TA et al (2017) Acute infarct extracellular volume mapping to quantify myocardial area at risk and chronic infarct size on cardiovascular magnetic resonance imaging. Circ Cardiovasc Imaging 10(7): $\mathrm{e} 006182$

13. Carrick D, Haig C, Rauhalammi S, Ahmed N, Mordi I, McEntegart M et al (2015) Prognostic significance of infarct core pathology revealed by quantitative non-contrast in comparison with contrast cardiac magnetic resonance imaging in reperfused ST-elevation myocardial infarction survivors. Eur Heart J 37(13):1044-1059

14. Carrick D, Haig C, Rauhalammi S, Ahmed N, Mordi I, McEntegart $\mathrm{M}$ et al (2015) Pathophysiology of LV remodeling in 
survivors of STEMI: inflammation, remote myocardium, and prognosis. JACC Cardiovasc Imaging 8(7):779-789

15. Reinstadler SJ, Stiermaier T, Liebetrau J, Fuernau G, Eitel C, de Waha $S$ et al (2018) Prognostic significance of remote myocardium alterations assessed by quantitative noncontrast $\mathrm{T} 1$ mapping in ST-segment elevation myocardial infarction. JACC Cardiovasc Imaging 11(3):411-419

16. Kidambi A, Motwani M, Uddin A, Ripley DP, McDiarmid AK, Swoboda PP et al (2017) Myocardial extracellular volume estimation by CMR predicts functional recovery following acute MI. JACC: Cardiovasc Imaging 10(9):989-999

17. Kidambi A, Mather AN, Swoboda P, Motwani M, Fairbairn TA, Greenwood JP et al (2013) Relationship between myocardial edema and regional myocardial function after reperfused acute myocardial infarction: an MR imaging study. Radiology 267(3):701-708

18. Yeon SB, Reichek N, Tallant BA, Lima JA, Calhoun LP, Clark NR et al (2001) Validation of in vivo myocardial strain measurement by magnetic resonance tagging with sonomicrometry. J Am Coll Cardiol 38(2):555-561

19. Augustine D, Lewandowski AJ, Lazdam M, Rai A, Francis J, Myerson S et al (2013) Global and regional left ventricular myocardial deformation measures by magnetic resonance feature tracking in healthy volunteers: comparison with tagging and relevance of gender. J Cardiovasc Magn Reson 15:8

20. Schuster A, Kutty S, Padiyath A, Parish V, Gribben P, Danford DA et al (2011) Cardiovascular magnetic resonance myocardial feature tracking detects quantitative wall motion during dobutamine stress. J Cardiovasc Magn Reson 13:58

21. Hor KN, Gottliebson WM, Carson C, Wash E, Cnota J, Fleck R et al (2010) Comparison of magnetic resonance feature tracking for strain calculation with harmonic phase imaging analysis. JACC Cardiovasc Imaging 3(2):144-151

22. Hor KN, Baumann R, Pedrizzetti G, Tonti G, Gottliebson WM, Taylor M et al (2011) Magnetic resonance derived myocardial strain assessment using feature tracking. J Vis Exp 48:2356

23. Eitel I, Stiermaier T, Lange T, Rommel K-P, Koschalka A, Kowallick JT et al (2018) Cardiac magnetic resonance myocardial feature tracking for optimized prediction of cardiovascular events following myocardial infarction. Cardiovasc Imaging: JACC 11:1433-1444

24. Dall'Armellina E, Piechnik S, Ferreira V, Si QL, Robson M, Francis J et al (2012) Cardiovascular magnetic resonance by non contrast T1-mapping allows assessment of severity of injury in acute myocardial infarction. J Cardiovasc Magn Reson 14(1):15

25. Piechnik SK, Ferreira VM, Dall'Armellina E, Cochlin LE, Greiser A, Neubauer S et al (2010) Shortened modified look-locker inversion recovery (ShMOLLI) for clinical myocardial T1-mapping at 1.5 and $3 \mathrm{~T}$ within a 9 heartbeat breathhold. J Cardiovasc Magn Reson 12:69

26. Ugander M, Bagi PS, Oki AJ, Chen B, Hsu L-Y, Aletras AH et al (2012) Myocardial edema as detected by pre-contrast T1 and T2
CMR delineates area at risk associated with acute myocardial infarction. JACC: Cardiovasc Imaging 5(6):596-603

27. Kali A, Choi EY, Sharif B, Kim YJ, Bi X, Spottiswoode B et al (2015) Native T1 mapping by 3-T CMR Imaging for characterization of chronic myocardial infarctions. JACC Cardiovasc Imaging 8(9):1019-1030

28. Eitel I, Desch S, Fuernau G, Hildebrand L, Gutberlet M, Schuler G et al (2010) Prognostic significance and determinants of myocardial salvage assessed by cardiovascular magnetic resonance in acute reperfused myocardial infarction. J Am Coll Cardiol 55(22):2470-2479

29. Khan JN, Singh A, Nazir SA, Kanagala P, Gershlick AH, McCann GP (2015) Comparison of cardiovascular magnetic resonance feature tracking and tagging for the assessment of left ventricular systolic strain in acute myocardial infarction. Eur J Radiol 84(5):840-848

30. Morton G, Schuster A, Jogiya R, Kutty S, Beerbaum P, Nagel E (2012) Inter-study reproducibility of cardiovascular magnetic resonance myocardial feature tracking. J Cardiovasc Magn Reson $14: 43$

31. Carberry J, Carrick D, Haig C, Rauhalammi SM, Ahmed N, Mordi I et al (2016) Remote zone extracellular volume and left ventricular remodeling in survivors of ST-elevation myocardial infarction. Hypertension 68(2):385-391

32. Fisher RA (1915) Frequency distribution of the values of the correlation coeffients in samples from an indefinitely large population. Biometrika 10(4):507-521

33. Piechnik SK, Ferreira VM, Lewandowski AJ, Ntusi NA, Banerjee R, Holloway C et al (2013) Normal variation of magnetic resonance $\mathrm{T} 1$ relaxation times in the human population at $1.5 \mathrm{~T}$ using ShMOLLI. J Cardiovasc Magn Reson 15(1):13

34. Buss SJ, Krautz B, Hofmann N, Sander Y, Rust L, Giusca S et al (2015) Prediction of functional recovery by cardiac magnetic resonance feature tracking imaging in first time ST-elevation myocardial infarction. Comparison to infarct size and transmurality by late gadolinium enhancement. Int J Cardiol 183:162-170

35. Dall'Armellina E, Karia N, Lindsay AC, Karamitsos TD, Ferreira V, Robson MD et al (2011) Dynamic changes of edema and late gadolinium enhancement after acute myocardial infarction and their relationship to functional recovery and salvage index. Circ Cardiovasc Imaging 4(3):228-236

36. Lee WW, Marinelli B, van der Laan AM, Sena BF, Gorbatov R, Leuschner F et al (2012) PET/MRI of inflammation in myocardial infarction. J Am Coll Cardiol 59(2):153-163

Publisher's Note Springer Nature remains neutral with regard to jurisdictional claims in published maps and institutional affiliations.

\title{
Affiliations
}

\author{
Malgorzata Wamil ${ }^{1,2} \cdot$ Alessandra Borlotti ${ }^{1,2} \cdot$ Dan Liu ${ }^{1,2} \cdot$ André Briosa e Gala ${ }^{1,2} \cdot$ Alessia Bracco ${ }^{1,2}$. \\ Mohammad Alkhaliil ${ }^{1,2}$. Giovanni Luigi De Maria ${ }^{1,2} \cdot$ Stefan K. Piechnik ${ }^{1,2} \cdot$ Vanessa M. Ferreira $^{1,2}$. \\ Adrian P. Banning ${ }^{1,2} \cdot$ Rajesh K. Kharbanda ${ }^{1,2} \cdot$ Stefan Neubauer ${ }^{1,2} \cdot$ Robin P. Choudhury $^{1,2} \cdot$ Keith M. Channon $^{1,2}$. \\ Erica Dall'Armellina ${ }^{3,4}$
}

Malgorzata Wamil

gosia.wamil@googlemail.com
Alessandra Borlotti

alessandra.borlotti@rdm.ox.ac.uk 
Dan Liu

danliu01@outlook.com

André Briosa e Gala

a.briosaegala@gmail.com

Alessia Bracco

alessia.bracco13@gmail.com

Mohammad Alkhalil

mohammad.alkhalil@cardiov.ox.ac.uk

Giovanni Luigi De Maria

gianluigidemaria@hotmail.it

Stefan K. Piechnik

stefan.piechnik@cardiov.ox.ac.uk

Vanessa M. Ferreira

Vanessa.ferreira@cardiov.ox.ac.uk

Adrian P. Banning

adrian.banning@ouh.nhs.uk

Rajesh K. Kharbanda

Rajesh.Kharbanda@ouh.nhs.uk
Stefan Neubauer

Stefan.neubauer@cardiov.ox.ac.uk

Robin P. Choudhury

robin.choudhury@cardiov.ox.ac.uk

Keith M. Channon

keith.channon@cardiov.ox.ac.uk

1 Division of Cardiovascular Medicine, Radcliffe Department of Medicine, John Radcliffe Hospital, University of Oxford, Oxford OX3 9DU, UK

2 NIHR Oxford Biomedical Research Centre, John Radcliffe Hospital, Oxford OX3 9DU, UK

3 Acute Vascular Imaging Centre, Radcliffe Department of Medicine, John Radcliffe Hospital, Oxford OX3 9DU, UK

4 The Leeds Institute of Cardiovascular and Metabolic Medicine, Division of Biomedical Imaging, University of Leeds, Leeds, UK 